\section{The value of otoscopy}

The need for a good general examination in all cases of trauma is well established, but in the region of the head and neck, examination of the ear canals and drums may be missed out when more dramatic, life-threatening injuries are sustained. This was demonstrated when a patient was admitted following an explosion in his micro-light aeroplane. The propeller was shattered, tearing off part of his left hand and embedding itself deeply into his skull and abdomen.

After resuscitation he underwent surgery under general anaesthesia by a combined team of plastic and neurosurgeons. The cranial incision extended from Dan to Beersheba, and the procedure involved resection of part of the cerebellum into which a section of the propeller was embedded.

Within a couple of weeks the patient had made a good recovery. He was left with unsteadiness, had a left-sided deafness and a left facial palsy for which a tarsorrhaphy was performed at a second operation. He later developed a discharge from the ear on the side of the impact and an opinion was sought from the ear nose and throat department. A foul-smelling discharge was aspirated under the microscope and antibiotic-steroid ear drops were administered. After a week the ear drum could be visualized more easily and what seemed like a piece of hard dried wax was seen fixed firmly up against the posterior wall of the canal. This was removed in the out-patient department and was found to be a piece of the propeller which had been

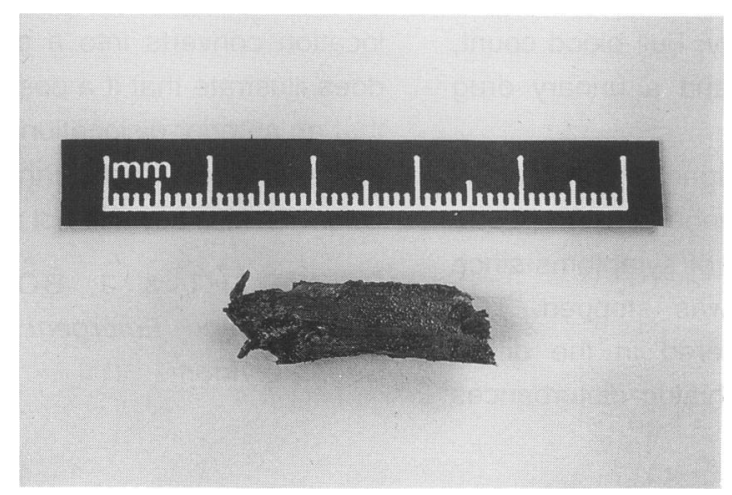

Fig. 1. Fragment of propeller that was removed from ear canal. driven into the canal (Fig. 1) and which was being seen head-on.

After further cleaning it was apparent that there were many further smaller splinters of wood. Further cleaning and removal of splinters under the microscope has been carried out in the out patient department without anaesthetic. A third anaesthetic was not thought to be warranted, although if the size and nature of the original piece had been suspected the patient would have been readmitted for this.

\section{J.D.C. BENNETT}

Lecturer in Otolaryngology, University of Manchester School of Medicine, Manchester

\section{Injuries sustained by passengers on buses}

Injuries sustained by passengers travelling on public buses are relatively uncommon. Public service vehicle passenger casualty rates, per billion passenger kilometre, are much lower than for cars, motor-cycles, pedal-cycles and pedestrians. ${ }^{1}$

Thirty injured bus passengers were identified over a 4-month period. Injuries sustained by the drivers and passengers when the bus was involved in a collision were excluded.

Of these 30 patients, 21 were female $(70 \%)$. The age range was from 2 to 81 years (mean age 56.8 years).

The majority of the injuries were minor, where the patients required reassurance and analgesia. However, there were two Colles fractures, one mid-shaft radius and ulna fracture, one fractured mid-shaft humerus, one supracondylar fracture of the right elbow, one fractured pelvis, one fractured rib, one fractured 5th metacarpal, two fractures of the nasal bone. The other injuries were mainly bruising and did not involve bony injury.

There were four admissions. One following abdominal injury (discharged the following day), one for fixation of the radius and ulna, one following fracture of the pelvis and a 68-year-old female who was so badly shaken that she was admitted for reassurance.

The majority of these injuries are preventable. A total of $50.3 \%$ occurred when the bus braked suddenly whilst passengers were waiting to alight, $20.3 \%$ occurred when the bus moved off quickly 